\title{
Potential Use of Radar Differential Reflectivity Measurements at Orthogonal Polarizations for Measuring Precipitation
}

\author{
T. A. Seliga and V. N. Bringi \\ Atmospheric Sciences Program and Department of Electrical Engineering, The Ohio State University, Columbus 43210
}

(Manuscript received 27 December 1974, in revised form 10 October 1975)

\begin{abstract}
The potential use of differential reflectivity measurements at orthogonal polarizations to determine rainfall rate is examined. The method involves measurements of $Z_{H}$ and $Z_{V}$, the radar reflectivity factors due to horizontally and vertically polarized incident waves respectively. The differential reflectivity, $Z_{D R}=10 \mathrm{log}$ $\left(Z_{H} / Z_{V}\right)$, which should be precisely determinate, occurs as a result of the distortion of raindrops as they fall at terminal velocity. The approximate theory of Gans for electromagnetic scattering by spheroids is applied to the distorted raindrops. Assuming a general exponential form for the raindrop size distribution, equations are derived relating the distribution parameters to the measurements. The determination of rainfall rate follows directly. Finally, the sensitivity of the distribution parameters to radar inaccuracies is examined, and several methods of implementing the measurements are suggested. It is concluded that good estimates of rainfall rate using a single non-attenuating wavelength radar are possible under ideal conditions.
\end{abstract}

\section{Introduction}

We wish to estimate the rainfall rate using a single non-attenuating wavelength radar without invoking any empirical relations between the radar reflectivity factor $(Z)$ and rainfall rate $(R)$. Atlas and Ulbrich (1974) have shown that such relations do not account for differing rainfall types; in addition, fluctuations about these relations are caused by a variety of natural processes such as drop size sorting, growth and evaporation. Atlas and Ulbrich have proposed a method where both $10 \mathrm{~cm}$ reflectivity and $3.2 \mathrm{~cm}$ attenuation measurements are used to determine $N_{0}$ and $D_{0}$ [parameters which describe the MarshallPalmer type exponential distribution, $N(D)=N_{0}$ $\left.\times \exp \left(-3.67 D / D_{0}\right)\right]$. Others, including Eccles and Atlas (1973) and Eccles and Mueller (1971) have proposed dual-wavelength schemes to extract meteorological information. Goldhirsh and Katz (1974) have examined the accuracies of such multiple-wavelength radar measurement schemes. It is evident that two characteristics of the precipitation structure have to be measured to determine the two-parameter distribution from which $R$ may be obtained. We propose to do this using differential and absolute reflectivity measurements at orthogonal polarizations and at nonattenuating wavelengths. The radar cross sections of raindrops (at orthogonal polarizations) are calculated using the theory of Gans (1912) for scattering from oblate spheriods, which are good approximations to the physical form of falling raindrops at terminal velocities.

\section{Theory}

Raindrops falling at terminal velocity are nonspherical. Pruppacher and Beard (1970) have shown that drop sizes up to an equivalent diameter of $3.0 \mathrm{~mm}$ may be assumed to be distorted into oblate spheriods. Larger drops assume a more complex shape. Warner and Hizal (1975) found that the complex shapes of actual raindrops as formulated by Pruppacher and Beard produce cross sections not very different from their equivalent oblate spheroids. In addition, Humphries (1974) finds that observations of microwave depolarizations are consistent with the idea that raindrops tend to fall as oblate spheriods with a vertical minor axis. Therefore, in this paper we will assume that all raindrops are distorted into oblate spheriods as they fall at their terminal velocity.

Actual and apparent canting of drop orientation from the vertical due to advection and radar elevation angle, respectively, are not expected to pose serious problems in making differential reflectivity measurements. Actual canting should be small in agreement with Humphries' conclusions about raindrop shape and motion. Measurements of backscattering cross sections for oblate spheroids by Atlas and Wexler (1963) and subsequent calculations by Warner and Hizal (1975) indicate that changes in cross section due to effective canting measured in the plane of incidence should be small for the range of drop sizes considered. Canting normal to the plane of incidence may, in fact, be measured by $Z_{D R}$, if the polarizations are jointly scanned in rotation; a mean 
canting angle of the drops in the scattering volume would be given by the rotation angle where this maximum $Z_{D R}$ occurs. Although this latter technique may prove useful for studying storm dynamics, consideration is restricted to determining the raindrop size distribution and rainfall rate.

A truncated, exponential raindrop size distribution of the form

$$
\begin{aligned}
N\left(D_{e q}\right)=N_{0} \exp \left(-3.67 D_{e q} / D_{0}\right) & {\left[\mathrm{m}^{-3} \mathrm{~cm}^{-1}\right], } \\
& 0<D_{e q} \leqslant 1.0 \mathrm{~cm},
\end{aligned}
$$

is assumed where $N_{0}$ and $D_{0}$ are parameters describing the magnitude and breadth of the distribution respectively; and $D_{e q}$ is the diameter of a spherical drop of volume equal to the volume of the actual drop. Experimental drop size distribution measurements by Blanchard (1953) and Cataneo (1968) have been found to be well approximated by this exponential form. except for very small sizes below about $1 \mathrm{~mm}$ diameter, In the calculations performed below, the difference between the actual and assumed distribution functions at these small drop sizes would have negligible effects on the results. For the Marshall-Palmer (1948) spectrum, the parameters have the values $N_{0}=8 \times 10^{4}$ $\mathrm{m}^{-3} \mathrm{~cm}^{-1}$ and $D_{0}=0.089 R^{0.22} \mathrm{~cm}$ where $R$ is in $\mathrm{mm} \mathrm{h}^{-1}$. In the present work $D_{0}$ and $R / N_{0}$ are related by the form $D_{0}=\left(c R / N_{0}\right)^{d}$, where $c$ and $d$ are constants. If the distribution (1) extends to drop diameters of all sizes, then $D_{0}$ becomes the median volume diameter (i.e., drops larger than $D_{0}$ contribute to half the total liquid water content per unit volume). Because of truncation in (1), $D_{0}$ loses its physical significance for $D_{0} \gtrsim 2.0 \mathrm{~mm}$. Pruppacher and Pitter (1971) believe that drops with $D_{e q} \gtrsim 1.0 \mathrm{~cm}$ will be hydrodynamically unstable and breakup would occur even if they fall in completely calm air. This provides the basis for the finite upper limit in (1).

The distortion of raindrops is given by the ratio $a / b$, where $a$ is the semi-minor axis and $b$ the semi-major axis of the ellipse that generates the oblate spheroid (see Fig. 1). The equations for $a / b$ are taken from

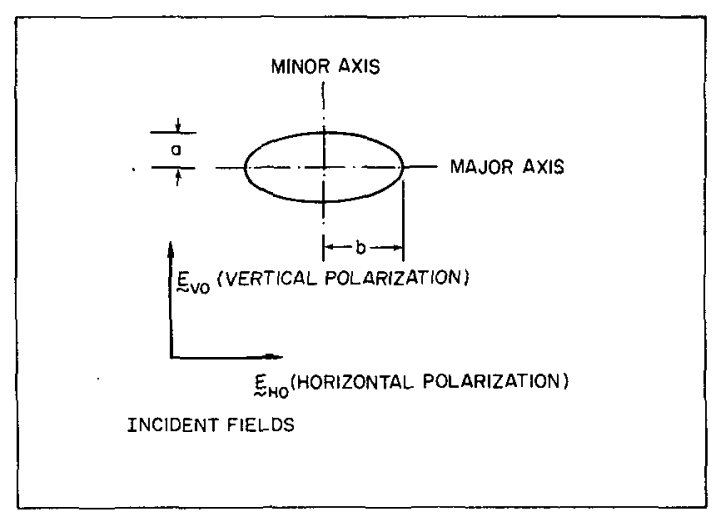

FIG. 1. An oblate spheroid is the body of revolution formed when an ellipse is rotated about its minor axis.
Pruppacher and Beard (1970):

$$
a / b=\left\{\begin{array}{lc}
1.0, & 0<D_{e q} \leqslant 0.028 \mathrm{~cm} \\
{\left[1-(9 / 32) D_{e q} \rho_{m} v^{2} / \mu\right]^{0.5},} & 0.028<D_{e q} \leqslant 0.1 \mathrm{~cm} \\
1.03-0.62 D_{e q}, & 0.1<D_{e q} \leqslant 1.0 \mathrm{~cm}
\end{array}\right.
$$

where $\rho_{m}=1.1937 \times 10^{-3} \mathrm{~g} \mathrm{~cm}^{-3}$ is the density of water vapor saturated air, $\mu=72.75$ ergs $\mathrm{cm}^{-2}$ is the surface tension of water as given by Pruppacher and Beard, and $v\left(\mathrm{~cm} \mathrm{~s}^{-1}\right)$ is the terminal velocity of the drop. In both equations, $D_{e q}$ is expressed in centimeters.

The radar cross section $\left(\sigma_{B}\right)$ of a spherical drop, assuming Rayleigh scattering, is given by (Stratton, 1941)

$$
\sigma_{B}=\left(\pi^{5} / \lambda^{4}\right)\left|\left(m^{2}-1\right) /\left(m^{2}+2\right)\right|^{2} D^{6} \quad\left[\mathrm{~cm}^{2}\right],
$$

where $\lambda$ is the wavelength, $m$ the refractive index of water, and $D$ the diameter of the drop. The reflectivity factor $Z$ is then expressed as

$$
Z=\int_{0}^{\infty} D^{6} N(D) d D \quad\left[\mathrm{~cm}^{6} \mathrm{~m}^{-3}\right],
$$

where $N(D)$ is the drop size distribution.

For our purposes the radar cross section of an oblate spheroid must be calculated. This is done following the theory of Gans (1912), whose work is essentially an extension of the Rayleigh theory for spheres applied to the case of oblate and prolate spheroids. Gans' theory has been outlined in Van De Hulst (1957) and used by Atlas et al. (1953) and Humphries (1974). We assume that measurements will be made at a non-attenuating wavelength (say $\lambda=10 \mathrm{~cm})$. For the largest drop $\left(D_{e q}=1.0 \mathrm{~cm}\right)$, the factor $2 \pi b / \lambda$ equals 0.42 . This factor is important since Mathur and Mueller (1955) have shown that Gans' theory is a good approximation for the range of sizes $(2 \pi b / \lambda)$ and distortions $(a / b)$ considered here. Their comparisons were, however, based upon an exact theory for perfectly conducting spheroids and may not apply here. More exact scattering theories such as those due to Waterman (1969) or Oguchi $(1960,1964)$ are preferred, but are not used here because of their complexity. However, the scattering cross sections of several sample drop sizes were calculated using Waterman's method as extended by Barber and Yeh (1974); these results also support Gans' theory for oblate spheroids in the size range of interest.

Let $\sigma_{H}$ and $\sigma_{V}$ be the horizontal and vertical radar cross sections of the oblate spheroid $\left(\sigma_{H}\right.$ refers to the radar cross section at horizontal polarization, $\sigma_{V}$ at vertical polarization). For a horizontally polarized incident wave, the scattered field is due to the induction of an electric dipole aligned along the major axis of the oblate spheroid (see Fig. 1). Similarly, a vertically polarized incident wave induces an electric 
dipole along the minor axis of the oblate spheroid. We also assume that the raindrops fall with zero canting angle, i.e., their minor axis remains vertical (Humphries, 1974).

Let $\mathbf{E}_{H 0}$ and $\mathbf{E}_{V 0}$ be the complex, plane wave electric field intensities incident on the oblate spheroid due to transmission of horizontal and vertical polarized waves respectively. The electric dipole moments induced in the oblate spheroid are given by

$$
\begin{aligned}
& \mathbf{p}_{H}=4 \pi \epsilon_{0} g^{\prime} \mathbf{E}_{H 0} \quad[\mathrm{C} \mathrm{m}], \\
& \mathbf{p}_{V}=4 \pi \epsilon_{0} g \mathbf{E}_{V 0} \quad[\mathrm{C} \mathrm{m}],
\end{aligned}
$$

where $\mathbf{p}_{H}$ and $\mathbf{p}_{V}$ are the dipole moments due to horizontal and vertical polarized incident waves. The factors $g$ and $g^{\prime}$ are given in Van De Hulst (1957):

$$
\begin{aligned}
& g=V\left(m^{2}-1\right) /\left[4 \pi+\left(m^{2}-1\right) P\right] \quad\left[\mathrm{m}^{3}\right], \\
& g^{\prime}=V\left(m^{2}-1\right) /\left[4 \pi+\left(m^{2}-1\right) P^{\prime}\right] \quad\left[\mathrm{m}^{3}\right],
\end{aligned}
$$

where $P$ and $P^{\prime}$ are geometrical factors defined below by (9) for oblate spheroids and $V$ is the volume of the drop:

$$
P=4 \pi-2 P^{\prime}=\left(4 \pi / e^{2}\right)\left\{1-[(a / b) / e] \sin ^{-1} e\right\} .
$$

Here $e$ is the eccentricity of the principal elliptical cross section $\left[e^{2}=1-(a / b)^{2}\right]$.

The magnitude of the far-zone, backscattered electric field intensities $\left(\mathbf{E}_{S H}\right.$ and $\left.\mathbf{E}_{S V}\right)$ due to dipole moments $p_{H}$ and $p_{V}$ are given by

$$
\begin{aligned}
& \left|\mathbf{E}_{S H}\right|=\left(\frac{1}{4} \pi \epsilon_{0}\right)\left(k^{2} / r\right)\left|\mathbf{p}_{H}\right| \quad\left[\mathrm{V} \mathrm{m}^{-1}\right], \\
& \left|\mathbf{E}_{S V}\right|=\left(\frac{1}{4} \pi \epsilon_{0}\right)\left(k^{2} / r\right)\left|\mathbf{p}_{V}\right| \quad\left[\mathrm{V} \mathrm{m}^{-1}\right],
\end{aligned}
$$

where $k$ is the free-space propagation constant $(k=2 \pi / \lambda)$ and $r$ the distance from the dipole to the field point. The horizontal and vertical radar cross sections are defined, respectively, by

$$
\begin{aligned}
& \sigma_{H}=\lim _{r \rightarrow \infty} 4 \pi r^{2}\left|\mathbf{E}_{S H}\right|^{2} /\left|\mathbf{E}_{H 0}\right|^{2} \quad\left[\mathrm{~m}^{2}\right], \\
& \sigma_{V}=\operatorname{Lim}_{r \rightarrow \infty} 4 \pi r^{2}\left|\mathbf{E}_{S V}\right|^{2} /\left|\mathbf{E}_{V 0}\right|^{2} \quad\left[\mathrm{~m}^{2}\right] .
\end{aligned}
$$

Using (5), (8), (9) and (10) in (12) we get

$$
\begin{array}{r}
\sigma_{H}=(16 / 9)\left(\pi^{7} / \lambda^{4}\right)\left(D_{e q}^{6}\right) \mid\left(m^{2}-1\right) / \\
{\left.\left[4 \pi+\left(m^{2}-1\right) P^{\prime}\right]\right|^{2}\left[\mathrm{~cm}^{2}\right],}
\end{array}
$$

where $P^{\prime}$ is defined in (9) and $\lambda$ and $D_{e q}$ are in centimeters. Similarly, $\sigma_{V}$ can be expressed as

$$
\begin{array}{r}
\sigma_{V}=(16 / 9)\left(\pi^{7} / \lambda^{4}\right)\left(D_{e q}^{6}\right) \mid\left(m^{2}-1\right) / \\
{\left.\left[4 \pi+\left(m^{2}-1\right) P\right]\right|^{2}\left[\mathrm{~cm}^{2}\right] .}
\end{array}
$$

The similarity of (14) and (15) to the Rayleigh cross section in (3) is evident. Either (14) or (15) reduces to (3) using $P=P^{\prime}=4 \pi / 3$ and $D_{e q}=D$.

The average backscattered power from a common pulse volume centered at a range $r$, due to horizontal or vertical polarized incident waves, may be expressed, respectively, by

$$
\begin{aligned}
& \left\langle P_{H}(r)\right\rangle=\left(C / r^{2}\right)\left[\int_{D_{e q}} \sigma_{H}\left(D_{e q}\right) N\left(D_{e q}\right) d D_{e q}\right][\mathrm{W}], \\
& \left\langle P_{V}(r)\right\rangle=\left(\mathrm{C} / r^{2}\right)\left[\int_{D_{e q}} \sigma_{V}\left(D_{e q}\right) N\left(D_{e q}\right) d D_{e q}\right][\mathrm{W}],
\end{aligned}
$$

where the angle braces denote a time-average and $C$ takes into account the radar constants. In order to express the scattered power in conventional form, we define $C^{*}$ such that

Then

$$
C^{*}=C(16 / 9)\left(\pi^{7} / \lambda^{4}\right) \text {. }
$$

$$
\begin{aligned}
& \left\langle P_{H}(r)\right\rangle=C^{*} Z_{H} / r^{2}, \\
& \left\langle P_{V}(r)\right\rangle=C^{*} Z_{V} / r^{2},
\end{aligned}
$$

where $Z_{H}$ and $Z_{V}$ are the horizontal and vertical radar reflectivity factors given by (21) and (22):

$Z_{H}=\int_{0}^{\infty} D_{e q}^{6}\left|\frac{m^{2}-1}{4 \pi+\left(m^{2}-1\right) P^{\prime}}\right|^{2} N\left(D_{e q}\right) d D_{e q}$

$\left[\mathrm{cm}^{6} \mathrm{~m}^{-3}\right]$

$Z_{V}=\int_{0}^{\infty} D_{e q}^{6}\left|\frac{m^{2}-1}{4 \pi+\left(m^{2}-1\right) P}\right|^{2} N\left(D_{e q}\right) d D_{e q}$

$\left[\mathrm{cm}^{6} \mathrm{~m}^{-3}\right]$.

Defining $S_{H}$ and $S_{V}$ as the horizontal and vertical shape functions,

$$
\begin{aligned}
& S_{H}(m, a / b)=\left|\left(m^{2}-1\right) /\left[4 \pi+\left(m^{2}-1\right) P^{\prime}\right]\right|^{2}, \\
& S_{V}(m, a / b)=\left|\left(m^{2}-1\right) /\left[4 \pi+\left(m^{2}-1\right) P\right]\right|^{2},
\end{aligned}
$$

and substituting (1) in (21) and (22), we obtain

$Z_{H} / N_{0}=\int_{0}^{1.0 \mathrm{~cm}} D_{e q}^{6} S_{H}(m, a / b) \exp \left(-3.67 D_{e q} / D_{0}\right) d D_{e q}$
$\left[\mathrm{~cm}^{6} \mathrm{~cm}^{-1}\right]$,

$Z_{V} / N_{0}=\int_{0}^{1.0 \mathrm{em}} D_{e q}^{6} S_{V}(m, a / b) \exp \left(-3.67 D_{e q} / D_{0}\right) d D_{e q}$

$\left[\mathrm{cm}^{6} \mathrm{~cm}^{-1}\right]$.

Differential reflectivity, defined as $Z_{D R}$ $=10 \log \left(Z_{H} / Z_{V}\right)$, is then given by

\section{$Z_{D R}=$}

$10 \log \left(\frac{\int_{0}^{1.0 \mathrm{~cm}} D_{e q}^{6} S_{H} \exp \left(-3.67 D_{e q} / D_{0}\right) d D_{e q}}{\int_{0}^{1.0 \mathrm{~cm}} D_{e q}^{6} S_{V} \exp \left(-3.67 D_{e q} / D_{0}\right) d D_{e q}}\right] \mathrm{dB}$. 


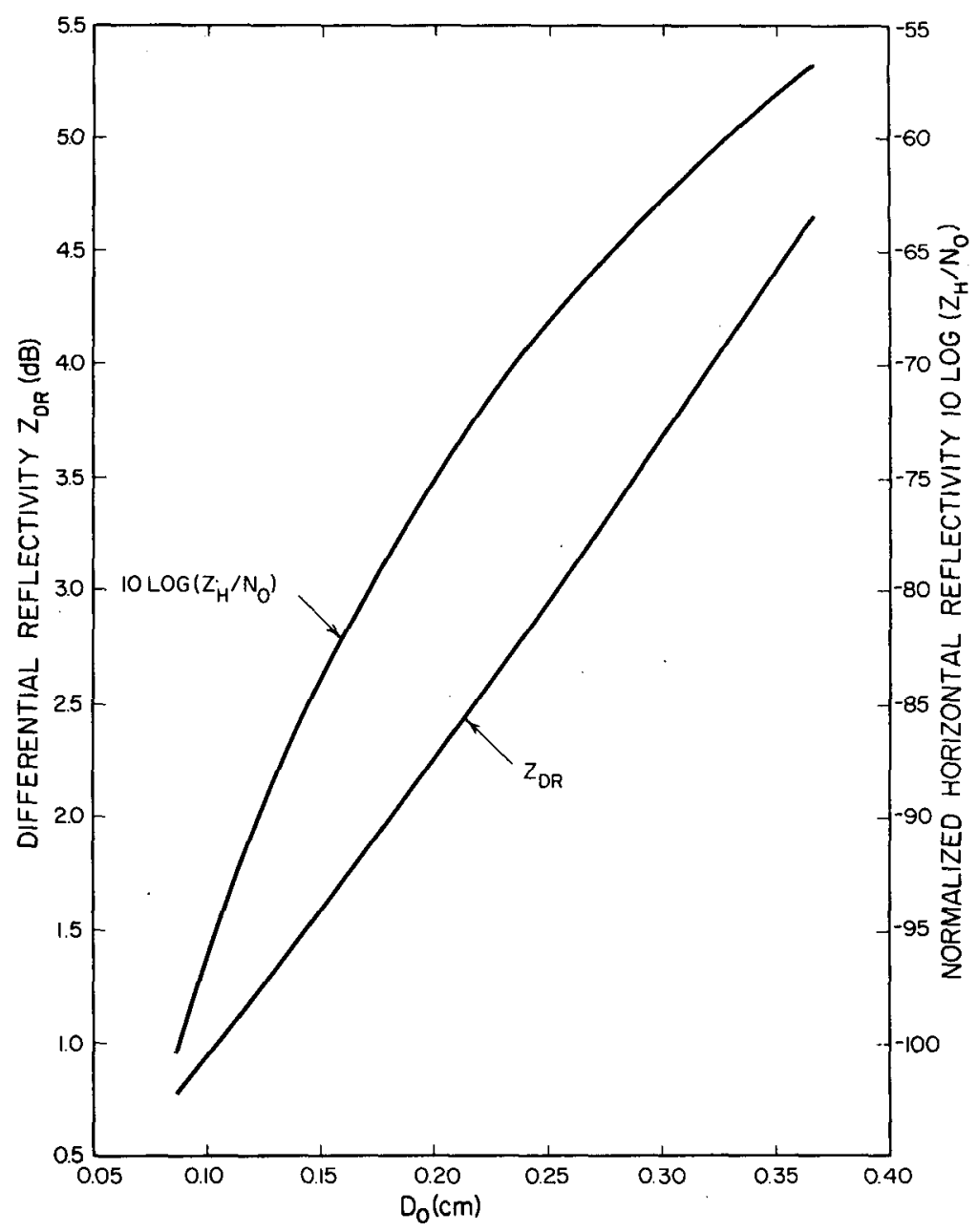

Fig. 2. Variations of $Z_{D R}$ and normalized horizontal reflectivity $10 \log \left(Z_{H} / N_{0}\right)$ with $D_{0}$.

It is noted that $Z_{D R}$ depends only on $D_{0}$ and is independent of the radar constants for equal system response at both polarizations. As shown in Section 5, $Z_{D R}$ should be precisely determined through relative power measurements, thereby yielding $D_{0}$ directly.

Finally, the rainfall rate $R\left(\mathrm{~mm} \mathrm{~h}^{-1}\right)$ can be expressed as

$$
\begin{array}{r}
R=0.6 \pi N_{0} \int_{0}^{1.0 \mathrm{em}} D_{e q}^{3} v\left(D_{e q}\right) \\
\quad \times \exp \left(-3.67 D_{e q} / D_{0}\right) d D_{e q}
\end{array}
$$

where $v\left(D_{e q}\right)$ is the terminal velocity of the drops $\left(\mathrm{m} \mathrm{s}^{-1}\right)$.

\section{Computations and analyses}

Each of the integrals in (25), (27) and (28) were divided into appropriate ranges according to (2) and the terminal velocity results of Gunn and Kinzer (1949). The drop distortion results given by Pruppacher and Beard (1970) were used. The integrals were numerically evaluated using a Gauss-Legendre quadrature method and are plotted as a function of $D_{0}$ in Figs. 2 and 3. The range for $D_{0}$ corresponds approximately to rainfall rates of 0.16 to $300 \mathrm{~mm} \mathrm{~h}^{-1}$, obtained by using the Marshall-Palmer empirical relation, $D_{0}=0.089 R^{0.22}[\mathrm{~cm}]$. It is clear that a determination of $Z_{D_{R}}$ through relative power measurements results in the determination of $D_{0}$ using the curve indicated in Fig. 2. Using this known value of $D_{0}$ and one of the measured absolute horizontal or vertical reflectivity factors, $Z_{H}$ or $Z_{V}, N_{0}$ can then be estimated from the normalized reflectivity curve given in Fig. 2. Knowing both $N_{0}$ and $D_{0}$, the rainfall rate $(R)$ is directly obtained from evaluation of (28) which is given in Fig. 3. As expected this reduces to a Marshall-Palmer type expression

$$
D_{0}=1.053\left(R / N_{0}\right)^{0.22} \quad[\mathrm{~cm}] .
$$




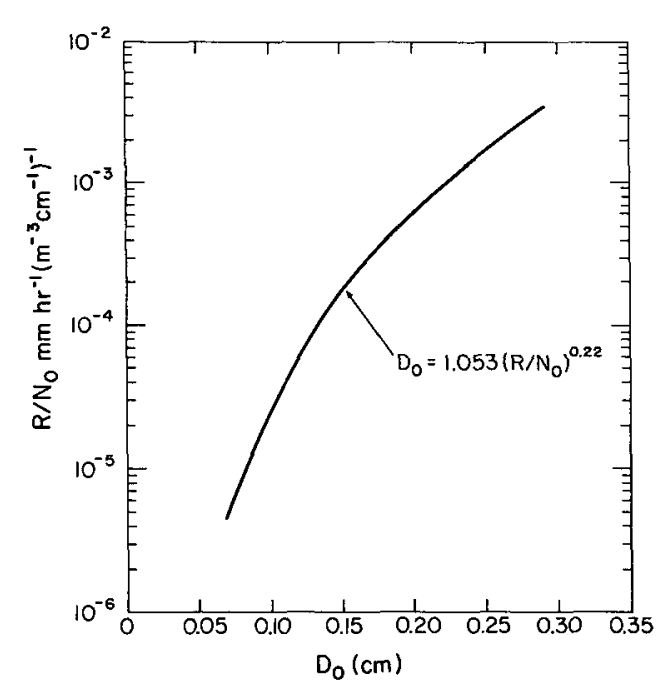

Frg. 3. Variation of $R / N_{0}$ as a function of $D_{0}$.

\section{Sensitivity of rainfall-rate distribution parameters to radar measurements}

We now wish to establish the uncertainties in $D_{0}$ and $R / N_{0}$ due to assumed errors in the measurement of $Z_{D R}[\mathrm{~dB}]$ and $Z_{H}$. In Fig. 4 we have plotted the uncertainty in $D_{0}$ resulting from assumed \pm 0.2 and $\pm 0.5 \mathrm{~dB}$ errors in the measurement of $Z_{D R}$. We feel these error estimates are reasonable, since $Z_{D R}$ is a differential measurement which should be easily and precisely determined (see Section 5). This occurs because $\sigma_{H}$ and $\sigma_{V}$ are precisely related to each other for each drop size in the scattering volume through the Pruppacher and Beard $(a / b)$ relationship [Eq. (2)]. Therefore, the standard deviation associated with the measurement of $Z_{D R}$ should be small. This is also important, since it implies that a single pulse measurement of $Z_{D R}$ is sufficient and that statistical averaging over a large number of independent radar returns should not be necessary.

The curves of Fig. 4 were obtained by adding the assumed errors to a series of $Z_{D R}$ values and establishing the corresponding values of $D_{0}$. Deviations from the mean $D_{0}$ values, $\delta D_{0}$, produce a corresponding uncertainty in establishing $R / N_{0}$. The ratios $R^{+} / N_{0}$ $\div R / N_{0}$ and $R^{-} / N_{0} \div R / N_{0}$ were then calculated and plotted as a function of $D_{0}$ and are shown in Fig. 5 . The superscripts correspond to the sign of the errors. Fig. 5 indicates the importance of measuring $Z_{D R}$ precisely. In the remaining error analysis we assume that $Z_{D R}$ can be measured to within an error of $\pm 0.2 \mathrm{~dB}$.

Fig. 5 shows that the uncertainty in establishing $R / N_{0}$ decreases as $D_{0}$ increases. For $D_{0}=0.1 \mathrm{~cm}$, $R^{+} / N_{0} \div R / N_{0}=1.9$, while $R^{-} / N_{0} \div R / N_{0}=0.48$. For larger $D_{0}$ 's $\left(D_{0} \gtrsim 0.2 \mathrm{~cm}\right)$ the corresponding ratios approach $R / N_{0}$ to within about $20 \%$.

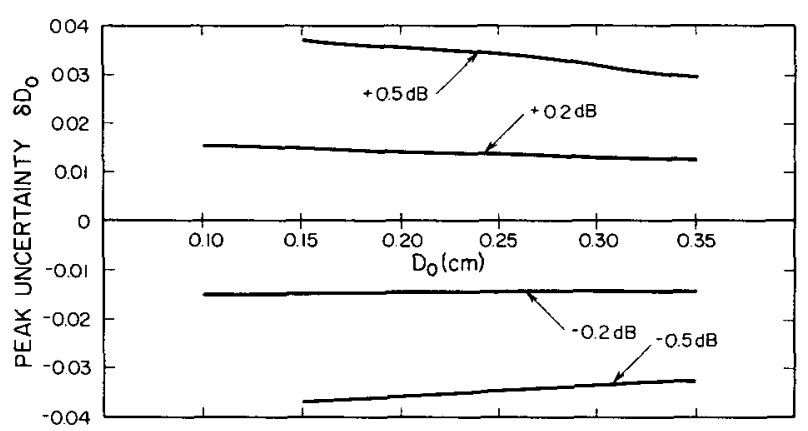

FIG. 4. Peak uncertainty in $D_{0}\left(\delta D_{0}\right)$ vs $D_{0}$ for \pm 0.2 and $\pm 0.5 \mathrm{~dB}$ measurement errors in $Z_{D R}$.

Next, the sensitivity of $R$ due to uncertainties in estimating $N_{0}$ is examined. $N_{0}$ is obtained from Fig. 2 and depends on knowing the values of both $D_{0}$ and $Z_{H}$. Therefore, the uncertainty in $N_{0}$ is due to the uncertainties in both these factors. Errors in $D_{0}$ produce corresponding errors in $10 \log \left(Z_{H} / N_{0}\right)$ as shown in Fig. 6. Measurement of the absolute horizontal reflectivity $Z_{H}$ depends on determining the radar constants which at best can be assumed known to within about $\pm 1.0 \mathrm{~dB}$. For example, this might be achieved with a radar which utilizes around 70 independent pulses to measure $Z_{H}$ (this gives a statistical error of about $\pm 0.5 \mathrm{~dB}$ with $90 \%$ confidence) and which has $\pm 0.5 \mathrm{~dB}$ error due to uncertainties in system calibration. The overall uncertainty in $R$ is compounded by the uncertainties in $D_{0}$ and $N_{0}$. For example, when $D_{0}=0.15 \mathrm{~cm}$, the overall uncertainty in $R$ is estimated to be within $\pm 5.7 \mathrm{~dB}$. However, the uncertainty decreases as $D_{0}$ increases. For $D_{0} \gtrsim 0.25 \mathrm{~cm}$, the uncertainty in $R$ is estimated to be within about $\pm 2.9 \mathrm{~dB}$.

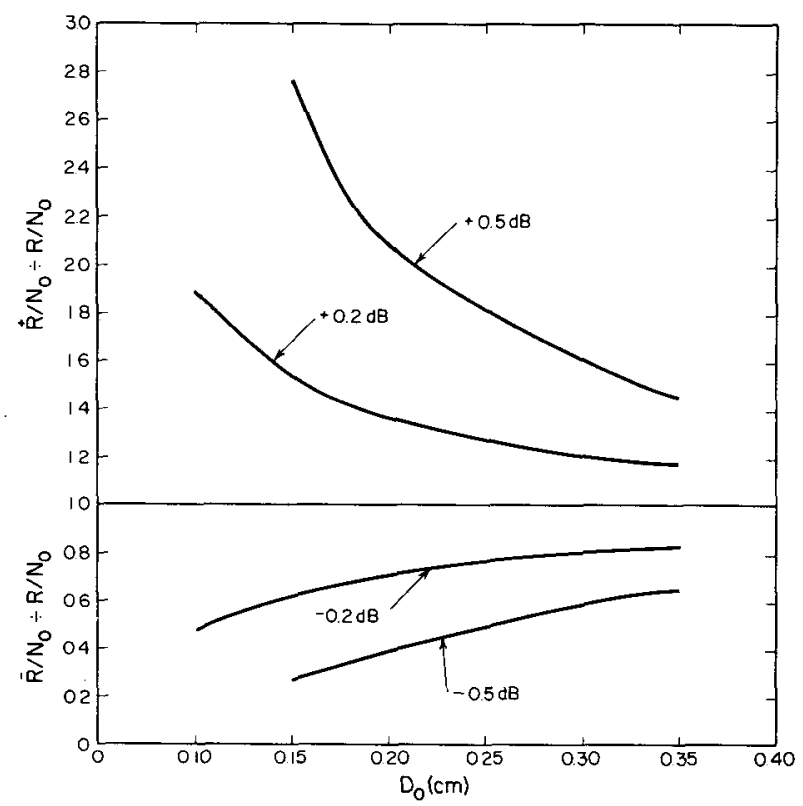

FIG. 5. Uncertainty in determining $R / N_{0}$ for $\pm 0.2 \mathrm{~dB}$ measurement errors in $Z_{D R}$. Positive superscript (on $R / N_{0}$ ) implies $\delta D_{0}$ is positive and vice versa. 


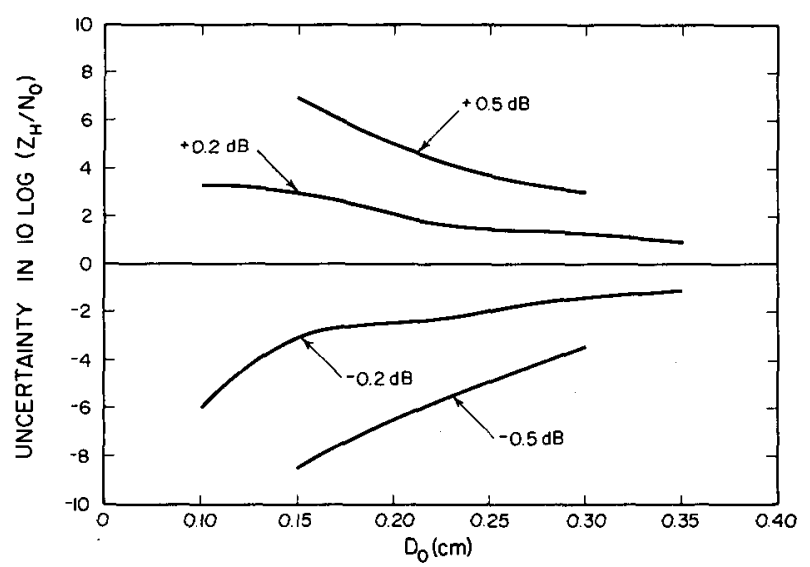

FIG. 6. Uncertainty in determining $10 \log \left(Z_{B} / N_{0}\right)$ for \pm 0.2 and $\pm 0.5 \mathrm{~dB}$ measurement errors in $Z_{D R}$.

It should be noted that the above analysis assumes that all errors compound and hence represents worstcase conditions.

If a second differentially measurable parameter (depending on $N_{0}$ ) is found, improved estimates of $R$ may be possible. In such a case, an error of $\pm 0.2 \mathrm{~dB}$ in this parameter would produce a maximum overall uncertainty in $R$ of about $\pm 2.1 \mathrm{~dB}\left(D_{0} \gtrsim 0.25 \mathrm{~cm}\right)$. This assumes that the sensitivity of this new parameter (relative to $N_{0}$ ) as a function of $D_{0}$ is not significantly different from that attributed to $10 \log \left(Z_{H} / N_{0}\right)$.

\section{Implementation of the measurement of $Z_{D R}$}

Many schemes to measure $Z_{D R}$ are possible with the exact choice dependent upon existing radar capabilities and economic factors. Nevertheless, it is appropriate here to suggest two methods which may prove feasible and easily adaptable to present systems. These are shown in Fig. 7. The block diagram of Fig. 7a shows a radar system which uses continuous sequential time-switching from horizontal to vertical polarizations. The transmitted radar pulse would alternately switch polarizations while the received signals would be passed through and detected by the same receiving system. Timing control would distinguish between the received horizontal and vertical polarized returns and select range-gates for continuous passage of the signal to narrow band-pass filters. Since a logarithmic receiver is used, $Z_{D R}$ would be the average

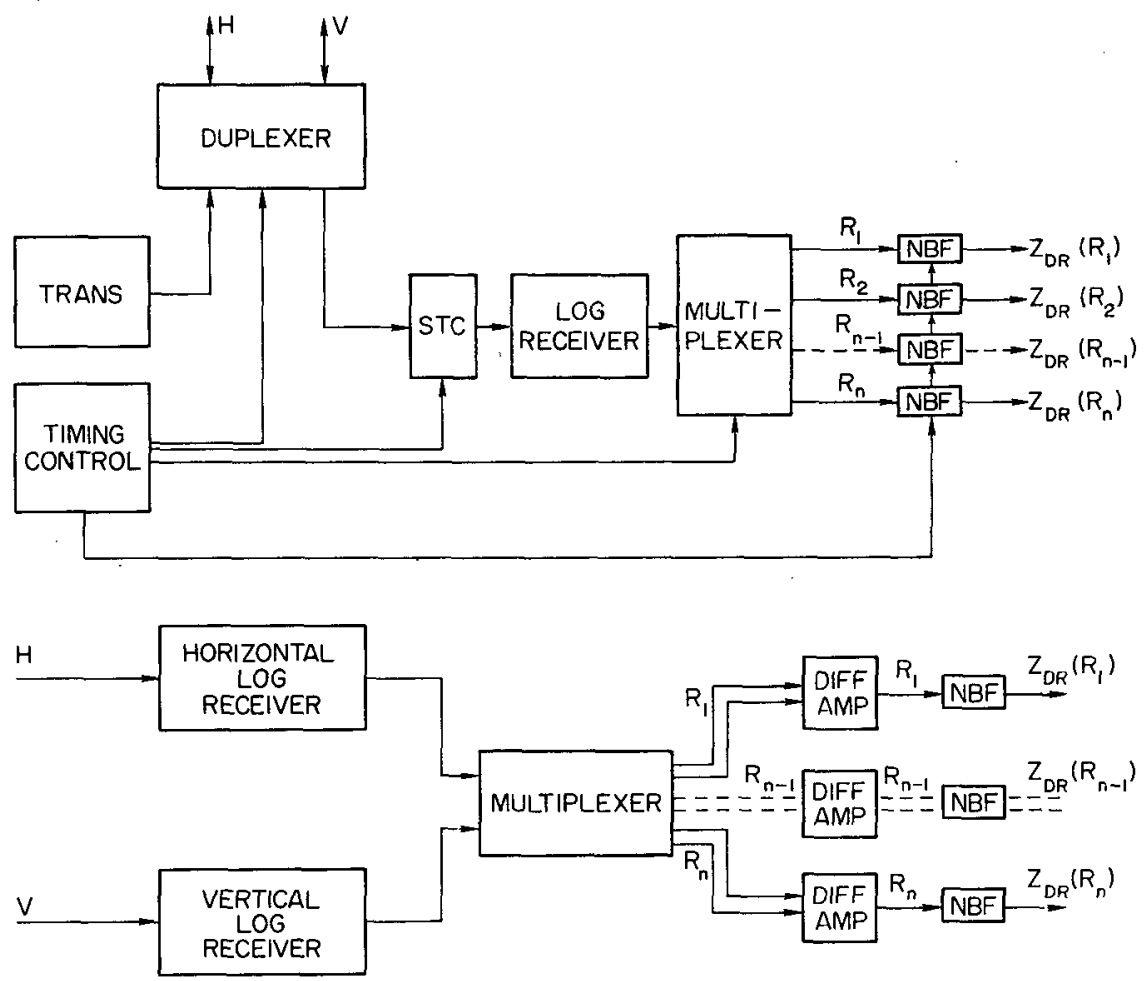

FIG. 7. Block diagram of radar system showing sequential or pulse-to-pulse technique for measuring $Z_{D R}$ [top (a)], and Radar receiving system for measuring $Z_{D R}$ when both horizontal and vertical polarizations are transmitted and received simultaneously [bottom (b)].

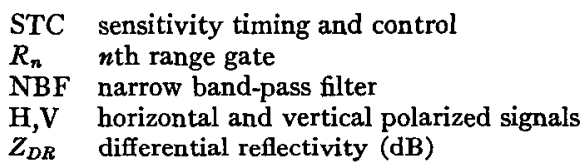


of the difference between the alternate amplitude (power) returns into the multiplexer and would be directly proportional to the output from the filters. The center frequency of the band-pass filters would be equal to one-half the pulse repetition frequency (PRF) of the transmitter - the entire system would operate similar to a system developed for D-region ionospheric studies using wave-interaction phenomena (e.g., see Weisbrod et al., 1964a, b).

The block diagram in Fig. 7b illustrates the receiving section of a radar which simultaneously transmits equal powers at both horizontal and vertical polarizations. This system requires two receivers from which the outputs are range-gated and differenced, the output of the narrow-band filter being $Z_{D R}(R)$. In this system the center frequency of the band-pass filter would be equal to the PRF. Cross-polarization coupling is not expected to seriously affect this technique, since the cross-polarized components are usually at least $20-30 \mathrm{~dB}$ below the main component while the greatest value for $Z_{D R}$ is about $4.5 \mathrm{~dB}$. Advantages and disadvantages of both these systems are presently under investigation by the authors.

\section{Conclusions}

This paper proposes a new method of radar measurement at non-attenuating wavelengths from which the raindrop size distribution parameters $\left(N_{0}, D_{0}\right)$ may be derived. Of particular importance is the use of differential reflectivity at orthogonal (horizontal and vertical) polarizations which gives $D_{0}$ directly, independent of $N_{0}$. The effect results from the distortion of raindrops into nearly oblate spheroids, oriented with their axis of revolution vertical. Model calculations were performed using the approximate scattering theory of Gans, the results of which indicate an ability to measure $D_{0}$ to within about $\pm 0.015 \mathrm{~cm}$ throughout the range $0.05 \leqslant D_{0} \leqslant 0.3 \mathrm{~cm}$ for $\pm 0.2 \mathrm{~dB}$ errors in the differential reflectivity $Z_{D R}$. Combining $Z_{D R}$ measurements with absolute reflectivity at either polarization (to within $\pm 1.0 \mathrm{~dB}$ ) produced an overall uncertainty in rain rate of about $\pm 5.7 \mathrm{~dB}$ for $D_{0}$ $\approx 0.15 \mathrm{~cm}$. The uncertainty was found to decrease with increasing $D_{0}$, resulting in a minimum of about $\pm 2.9 \mathrm{~dB}$ for $D_{0} \gtrsim 0.25 \mathrm{~cm}$ under worst-case conditions.

Further improvements in using radar to determine rain rates may result by combining other differential measurements with $Z_{D R}$. These might include differential phase shifts or attenuation along the propagation path. These concepts are suggested by the work of Oguchi and Hosoya (1974) and are presently under investigation. Other possible applications of the method may be the use of $Z_{D R}$ to determine accurately $D_{0}$ which by itself may be a useful parameter for classifying precipitation, or which with ground truth measurements may be used to parameterize $Z_{D R}-R$ relationships, similar to $Z-R$ relationships obtained by many others (e.g., see Borovikov et al., 1967; Neiburger and Weickmann, 1974).

Acknowledgments. This research was performed in part through the support of an Ohio State University Graduate Research Associateship through its Atmospheric Sciences Program and a University Corporation of Atmospheric Research Fellowship to V. N. Bringi. Discussions and criticisms of colleagues in the Department of Electrical Engineering are greatly appreciated.

\section{REFERENCES}

Atlas, D., and C. W, Ulbrich, 1974: The physical basis for attenuation-rainfall relationships and the measurement of rainfall parameters by combined attenuation and radar methods. J. Rech. Almos., 8, 275-298.

-, M. Kerker and W. Hitschfield, 1953: Scattering and attenuation by non-spherical atmospheric particles. J. Atmos. Terr. Phys., 3, 108-109.

- , and R. Wexler, 1963 : Back-scatter by oblate ice spheroids. J. Atmos. Sci., 20, 48-61.

Barber, P., and C. Yeh, 1974: Scattering of electromagnetic waves by arbitrarily shaped dielectric bodies. Tech. Rept., UCLA-ENG-7431, Electrical Sciences and Engineering Dept., University of California, Los Angeles.

Blanchard, D., 1953: Raindrop size distribution in Hawaiian rains. J. Meteor., 10, 457-473.

Borovikov, A. M., V. V. Kostarev, I. P. Mazin, V. I. Smirnov and A. A. Chernikov, 1967: Radar Measurement of Precipitation Rate. Israel Program for Scientific Translations, Jerusalem, 1970.

Cataneo, R., 1968: A comparison of raindrop size spectra between Miami, Florida and Corvallis, Oregon. Trans. Ill. Acad. Sci., 61, $165-170$.

Eccles, P. J., and D. Atlas, 1973: A dual-wavelength radar hail detector. J. A ppl. Meteor., 5, 847-854.

- , and E. A. Mueller, 1971: X-band attenuation and liquid water content estimation by a dual-wavelength radar. $J$. Appl. Meteor., 6, 1252-1259.

Gans, R., 1912 : Uber die Form ultramikroskopischer Goldteilchen. Ann. Phys., 37, 881-900.

Goldhirsh, J., and I. Katz, 1974: Estimation of raindrop size distribution using multiple wavelength radar systems. Radio Sci., 4, 439-446.

Gunn, R., and G. D. Kinzer, 1949: The terminal velocity of fall for water droplets in stagnant air. J. Meleor., 6, 243-248.

Humphries, R. G., 1974: Depolarization effects at $3 \mathrm{GHz}$ due to precipitation. Sci. Rept. MW-82, Stormy Weather Group, McGill University.

Marshall, J. S., and W. M. K. Palmer, 1948: The distribution of raindrops with size. J. Meteor., 5, 165-166.

Mathur, P. N., and E. A. Mueller, 1955: Radar back-scattering cross-sections from non-spherical drops. Preprints 5 th Weather Radar Conf., Asbury Park, N. J., Amer. Meteor. Soc., 269-273.

Neiburger, M., and H. K. Weickmann, 1974: Weather and Climate Modification, Chap. 3. Wiley, 842 pp.

Oguchi, T., 1960: Attenuation of electromagnetic wave due to rain with distorted raindrops. J. Radio Res. Lab., 7, 467-485.

- 1964 : Attenuation of electromagnetic wave due to rain with distorted raindrops (Part II). J. Radio Res. Lab., 11, $19-43$.

- , and Y. Hosoya, 1974: Differential attenuation and differential phase shift of radio waves due to rain: Calculations of microwave and millimeter wave regions. J. Rech. Atmos., 8, 121-128. 
Pruppacher, H. R., and K. V. Beard, 1970: A wind tunnel investigation of the internal circulation and shape of water drops falling at terminal velocity in air. Quart. $J$. Roy. Meteor. Soc., 96, 247-256.

- , and R. L. Pitter, 1971: A semi-empirical determination of the shape of cloud and raindrops. J. Atmos. Sci., 28, 86-94.

Stratton, J. A., 1941 : Electromagnetic Theory. McGraw Hill, $615 \mathrm{pp}$.

Van De Hulst, H. C., 1957: Light Scattering by Small Particles. Wiley, $470 \mathrm{pp}$.
Warner, C., and A. Hizal, 1975 : Scattering and depolarization of microwaves by spheroidal raindrops. (Submitted to Proc. $I E E$.)

Waterman, P. C., 1969: Scattering by dielectric obstacles. Alta Freq., 38 (speciale), 348-352.

Weisbrod, S., A. J. Ferraro and H. S. Lee, 1964a: Investigation of phase interaction as a means of studying the lower ionosphere. J. Geophys. Res., 69, 2337-2347.

$\longrightarrow$, H. S. Lee and A. J. Ferraro, 1964b: Preliminary measurements of radio wave phase interaction. J. Geophys. Res., 69, 1448-1449. 